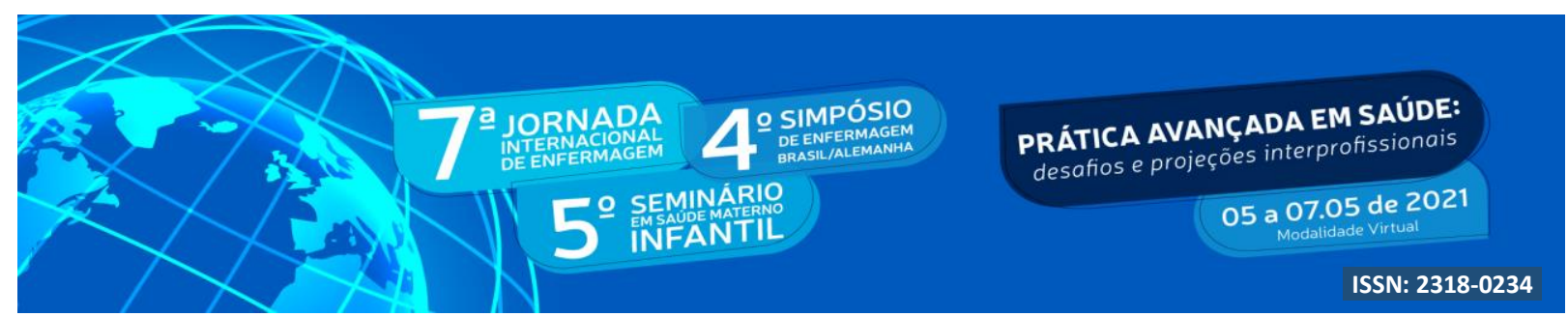

DOI: http://doi.org/10.48195/jie2021-160

\title{
DESAFIOS DOS ENFERMEIROS NO TRATAMENTO DE LESÕES DE PELE: REVISÃO NARRATIVA
}

\section{Adriana Dall'Asta Pereira; Carla Lizandra de Lima Ferreira; Caroline Colares; Renata Rodrigues da Silva.}

\begin{abstract}
RESUMO
O objetivo desse estudo foi analisar na produção cientifica, quais as barreiras encontradas pelos enfermeiros na hora da escolha da melhor conduta no tratamento de lesões. Trata-se de uma revisão narrativa de literatura nas bases de dados da Literatura Latino-Americana e do Caribe em Ciências da Saúde (LILACS), Base de dados de enfermagem (BDENF). A coleta de dados correspondeu aos meses de outubro a novembro de 2020. Como resultados emergiu a necessidade de atualização e aperfeiçoamento dos profissionais enfermeiros para realizar cuidado e a escolha adequada da cobertura. Conclui-se que os estudos apresentaram que dentre os desafios na atuação do enfermeiro, destaca-se a necessidade de atualização e aperfeiçoamento dos profissionais para realizar cuidado e a escolha adequada da cobertura. Enfermeiro possui habilidades e competências técnico - cientificas para exercer o cuidado com lesões, tendo um papel de grande importância para garantia de um cuidado integral do indivíduo.
\end{abstract}

Palavras-chave: Enfermeiros (as); Cuidados de Enfermagem; Lesões.

\begin{abstract}
This study was to analyze in the scientific production, which are the barriers found by nurses when choosing the best conduct in the treatment of injuries. It is a narrative review of literature in the databases of Latin American and Caribbean Literature in Health Sciences (LILACS), Nursing database (BDENF). Data collection corresponded to the months of October to November of the current year. As a result of the careful thematic analysis that resulted Need for updating and improvement of professional nurses to provide care and the appropriate choice of coverage. It is concluded that the studies showed that among the challenges in the nurse's performance, the need to update and improve the professionals to perform care and the appropriate choice of coverage stands out. Due to its articulating and integrating character, the Nurse has technical and scientific skills and competences to exercise care with injuries, playing a very important role in guaranteeing comprehensive care for the individual and performs an extremely relevant job in the case of wounds, since it has greater contact with the patient.
\end{abstract}

Key Words: Nurse; Nursing care; Injuries. 


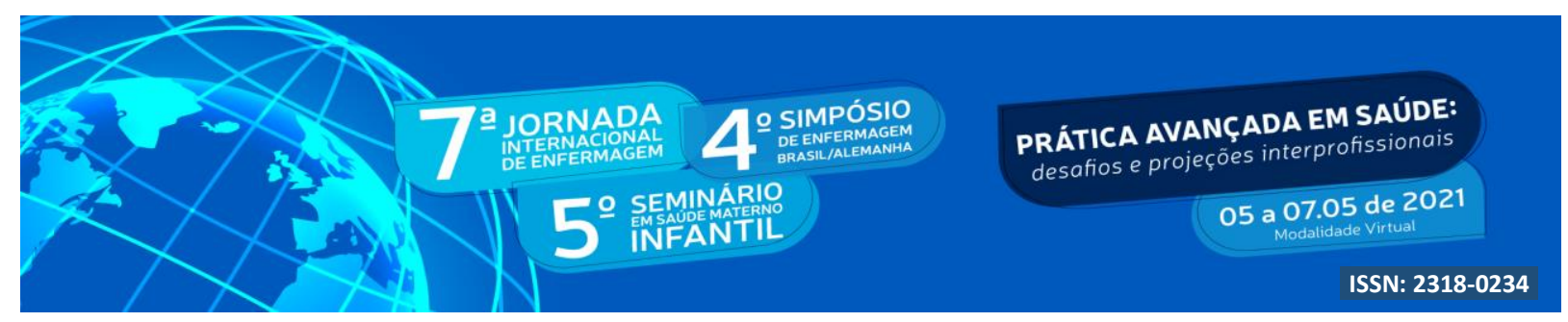

\section{INTRODUÇÃO}

Dentro do ambiente hospitalar existem vários assuntos a serem pesquisados e discutidos sobre os cuidados prestados ao paciente, entre eles pode-se destacar que o tema sobre lesões e curativos fazem parte da assistência prestada pelo enfermeiro. As feridas acometem a população geral, constituindo um sério problema de saúde pública, pois determina um alto índice de pessoas com alterações na integridade da pele. No Brasil, aproximadamente 3\% da população do país têm algum tipo de lesão, sendo que esse percentual aumenta para $10 \%$ entre as pessoas com Diabetes Mellitus, apresentando lesões crônicas (OLIVEIRA et al.,2016).

Pacientes com lesões, principalmente as que se caracterizam como crônicas, são um desafio para os profissionais da saúde em sua prática clínica, pois elas causam dor, imobilidade, incapacidade, alterações psicológicas e emocionais relacionadas à autoestima e à autoimagem determinando modificações em seus cotidianos ocorridos devido sua hospitalização e isolamento social (FERNANDA et all. 2018). Nesse sentido, o conhecimento sobre lesões de pele é de extrema importância para que durante o exame físico, a avaliação seja realizada com coerência, conhecimento e responsabilidade do profissional, e também para ser repassado para a equipe de enfermagem.

A Resolução do Conselho Federal de Enfermagem 501/2015, atribui ao enfermeiro o cuidado de lesões. O Enfermeiro pode realizar a consulta de Enfermagem, prescrever e executar curativo, coordenar e supervisionar a equipe de Enfermagem na prevenção e cuidados de feridas e no registro da evolução da ferida, dentre outras atribuições específicas (COFEN, 2015).O enfermeiro é responsável pelo planejamento, organização, execução e avaliação contínua da assistência realizada aos pacientes com lesão crônica apontando estratégias de cuidado seguro com a pele e de qualidade por meio da Sistematização da Assistência de Enfermagem (SAE)( SANTOS,2014).

Como o profissional de enfermagem está diretamente relacionado ao tratamento de feridas, deve resgatar a responsabilidade de manter a observação intensiva com relação aos fatores locais, sistêmicos e externos que condicionam o surgimento da ferida ou interfiram no processo de cicatrização. Para tanto, é necessária uma visão clínica que relacione alguns pontos importantes que influenciam neste processo, como o controle da patologia de base, aspectos nutricionais, infecciosos, medicamentosos e o rigor e a qualidade do cuidado educativo. A adequada avaliação possibilita a adoção de condutas a fim de interferir nas situações que possam ser prejudiciais e deste modo, permitir o alcance da completa cicatrização (ABBADE, 2010). Para uma avaliação integral do indivíduo, não somente da ferida, é fundamental a utilização de instrumentos que lhes fornecerão subsídios no 


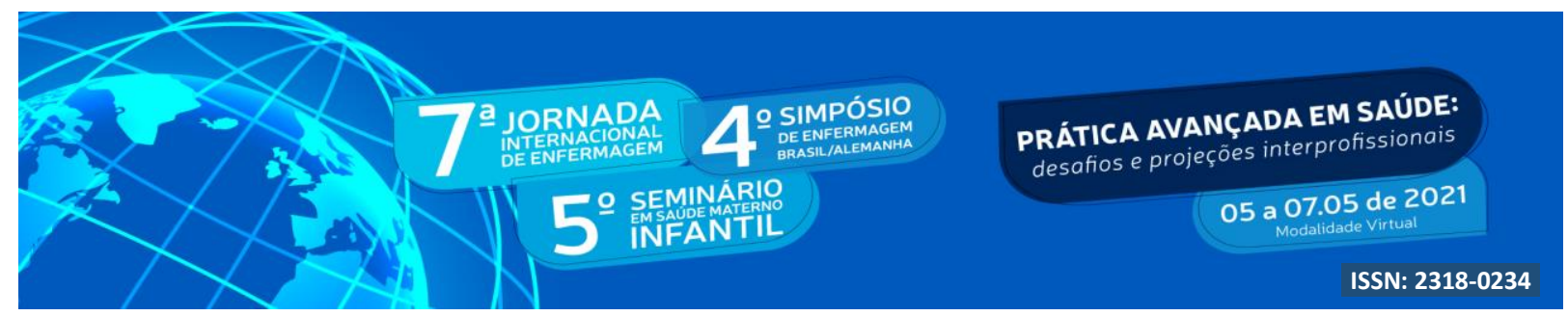

tratamento de feridas e a escolha do agente terapêutico adequado, além de possibilitar o acompanhamento da evolução da lesão durante todo o processo de reparação tecidual em cada fase da cicatrização (ESPÍRITO SANTO et all.,2013).

Diante do exposto, o estudo partirá da questão de pesquisa: quais as dificuldades que os enfermeiros têm para avaliar e tratar as lesões de pele dos pacientes?

\section{OBJETIVO}

Este estudo tem como objetivo analisar na produção cientifica, quais as barreiras encontradas pelos enfermeiros na hora da escolha da melhor conduta no tratamento de lesões.

\section{METODOLOGIA}

O presente trabalho trata-se de uma revisão Narrativa da literatura, a qual sintetiza resultados das pesquisas anteriormente realizadas e aponta as conclusões do corpus da literatura sobre um fenômeno específico, compreendendo todos os estudos ligados à questão norteadora que orienta a busca desta literatura (MENDES; SILVEIRA; GALVÃO, 2008).

Caracteriza-se por ser uma pesquisa ampla e que geralmente parte de uma temática mais aberta. Nesse tipo de estudo, os dados, após resumidos e comparados, permitem a obtenção de conclusões gerais sobre o problema da pesquisa. Deve-se seguir um processo de análise que possibilite identificar as lacunas do conhecimento em relação ao fenômeno em estudo, identificar a necessidade de futuras pesquisas, revelar as questões centrais da área em estudo, identificar marcos conceitual ou teórico, assim como mostrar o estado da arte da produção científico como resultados de estudos sobre um tema (CROSSETTI, 2012).

A busca das produções científicas foi realizada nas bases de dados de Enfermagem (BDENF) e Base de dados Latino- Americano e do Caribe em Ciências da Saúde (LILACS). Foram utilizados os seguintes descritores: "CUIDADOS DE ENFERMAGEM" and "LESÕES" or "FERIDAS". Quanto a questão norteadora de pesquisa, buscou-se responder: Quais as barreiras enfrentadas pelos Enfermeiros na melhor escolha para o tratamento de lesões de pele?

Os critérios de inclusão foram trabalhos completos que abordassem a temática, disponíveis on-line na íntegra de forma gratuita, publicados nos últimos cinco anos, no qual apresentam produções mais atualizadas a respeito do tema e disponíveis em língua portuguesa 


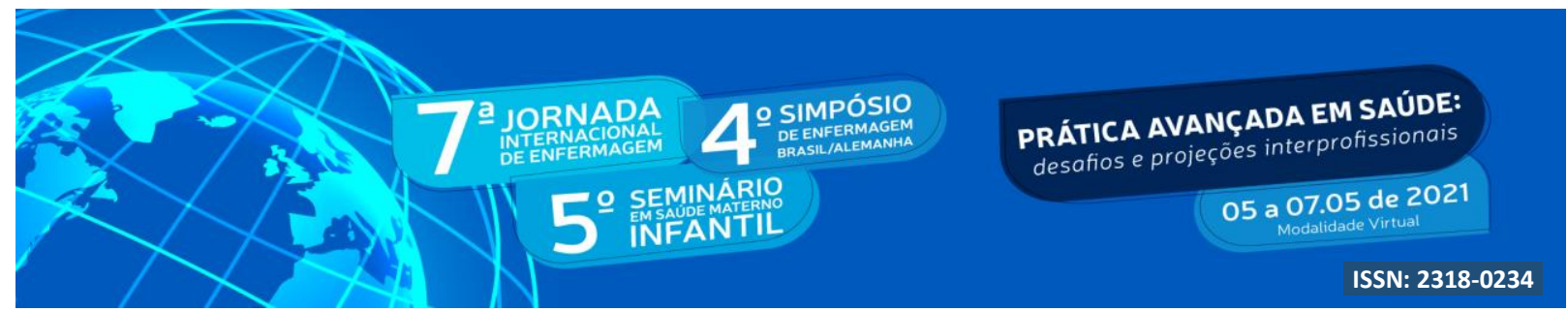

e inglesa. Os critérios de exclusão foram artigos sem resumo na base de dados ou incompletos, artigos repetidos nas bases de dados pesquisadas e que não atendessem o objetivo proposto neste trabalho.

Em primeiro momento, foi realizado a pesquisa e o refinamento das informações nas bases de dados que totalizaram 557 artigos na LILACS, na base de dados BDENF 494 artigos. A partir dos critérios de inclusão utilizados, permaneceram somente seis artigos, sendo três da base de dados LILACS três da base de dados BDENF.

Em segundo momento foram extraídos os dados dos artigos selecionados e os mesmos foram expostos em quadro sinóptico a fim de simplificar a identificação de suas informações. Em terceiro momento foi realizada a análise temática criteriosa que resultaram duas categorias temáticas (MINAYO, 2016). O aspecto ético da presente revisão respeitou a Lei 9.610/98 no intuito de preservar as ideias, os conceitos e as definições dos autores dos artigos analisados. (BRASIL, 1998).

\section{RESULTADOS E DISCUSSÃO}

Ao caracterizar os seis artigos, ressalta-se que neles os autores informaram sua especialidade, sendo todos enfermeiros. Quanto à metodologia os artigos encontrados três caracterizam por serem qualitativos e três por serem descritivo exploratórios. Quanto à base de dados de pesquisa caracteriza-se que três artigos estão disponíveis na fonte de pesquisa do LILACS, três na BDENF. Para a síntese dos artigos formou-se o quadro sinóptico (Quadro 1) que compõem o corpus da pesquisa de revisão narrativa no qual constam: letra do artigo, referência do artigo, objetivos, método e principais resultados.

Quadro 1 - Corpus da pesquisa de revisão narrativa.

\begin{tabular}{|l|l|l|l|l|}
\hline \multicolumn{5}{|c|}{ ARTIGOS SELECIONADOS } \\
\hline Artigo & Referência & Objetivos & Método & Principais resultados \\
\hline
\end{tabular}




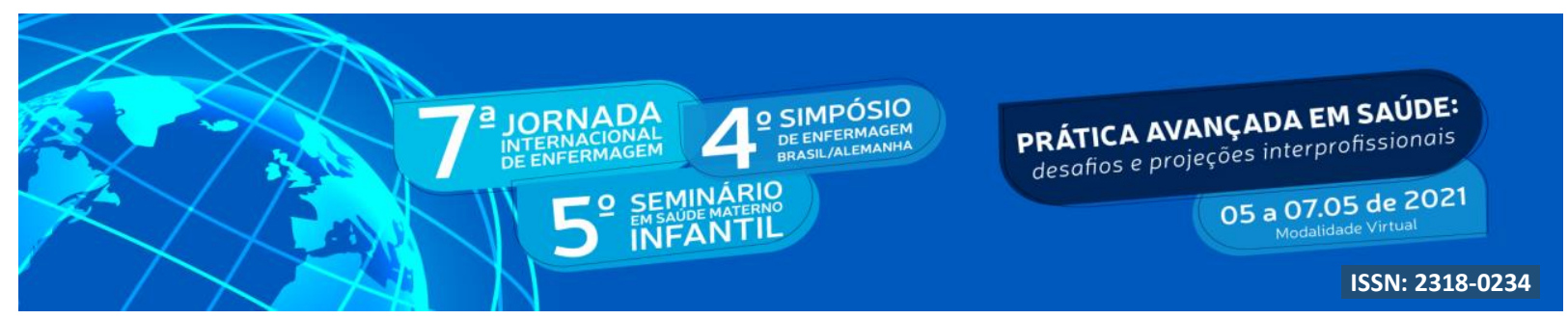

\begin{tabular}{|c|c|c|c|c|}
\hline $\mathbf{A}$ & $\begin{array}{l}\text { BANDEIRA, } \\
\text { A.G., } \\
\text { BIELEMANN, } \\
\text { V.L.M., GALLO } \\
\text { C.M.C., } \\
\text { CASARIN, S.T. }\end{array}$ & $\begin{array}{l}\text { Identificar os limites e as } \\
\text { possibilidades encontradas } \\
\text { pelas famílias frente ao } \\
\text { cuidado de feridas a } \\
\text { domicílio de clientes } \\
\text { acompanhados ar } \\
\text { profissionais de um Grupo } \\
\text { de Curativos. }\end{array}$ & $\begin{array}{l}\text { Qualitativo, } \\
\text { descritivo e } \\
\text { exploratório. }\end{array}$ & $\begin{array}{l}\text { O que se destacou foi o apoio } \\
\text { mútuo entre os membros do } \\
\text { grupo familiar, ao estar em casa } \\
\text { realizando os cuidados e por } \\
\text { disponibilizarem de um serviço } \\
\text { de referência e de confiança } \\
\text { para o tratamento de feridas. }\end{array}$ \\
\hline B & $\begin{array}{l}\text { CAUDURO, F.P., } \\
\text { SCHNEIDER,, } \\
\text { S.M.B. } \\
\text { MENEGON, D.B., } \\
\text { DUARTE, E.R.M., } \\
\text { PAZ, P.O., } \\
\text { KAISER DE. }\end{array}$ & $\begin{array}{l}\text { Conhecer a atuação dos } \\
\text { Enfermeiros no cuidado aos } \\
\text { pacientes com lesão de } \\
\text { pele. }\end{array}$ & $\begin{array}{l}\text { Qualitativo, } \\
\text { descritivo e } \\
\text { exploratório. }\end{array}$ & $\begin{array}{l}\text { Considerou-se aprimoramento } \\
\text { do Enfermeiro através da } \\
\text { educação permanente, pelo } \\
\text { trabalho em equipe e pelo } \\
\text { auxílio da Comissão de } \\
\text { Prevenção e tratamento de } \\
\text { Feridas. }\end{array}$ \\
\hline $\mathbf{C}$ & $\begin{array}{l}\text { SOUZA, } \\
\text { RODRIGUES, } \\
\text { N.H., } \\
\text { L.GA, } \\
\text { DM, } \\
\text { SILVA, } \\
\text { SG, SOUZA L LM. }\end{array}$ & $\begin{array}{l}\text { Conhecer como os } \\
\text { Enfermeiros atuam na } \\
\text { avaliação e tratamento de } \\
\text { lesões por pressão no } \\
\text { contexto da Estratégia } \\
\text { Saúde da Família. }\end{array}$ & $\begin{array}{l}\text { Qualitativo, } \\
\text { descritivo e } \\
\text { exploratório. }\end{array}$ & $\begin{array}{l}\text { O Enfermeiro necessita possuir } \\
\text { o conhecimento teórico-prático } \\
\text { para que, juntamente à equipe } \\
\text { multiprofissional e à família, se } \\
\text { promova o cuidado na } \\
\text { prevenção e tratamento } \\
\text { necessário a estes pacientes } \\
\text { portadores de lesões de pele. }\end{array}$ \\
\hline D & $\begin{array}{lr}\text { FARIA, } & \text { GBG, } \\
\text { PRADO } & \text { TN, } \\
\text { LIMA } & \text { EFA, } \\
\text { REGENSKI } & \text { MB, } \\
\text { BORGHARDT } \\
\text { AT, MASSARONI } \\
\text { L. }\end{array}$ & $\begin{array}{l}\text { Avaliar o conhecimento dos } \\
\text { Enfermeiros sobre a } \\
\text { avaliação e tratamento de } \\
\text { feridas e descrever a prática } \\
\text { clínica no cuidado com } \\
\text { feridas. }\end{array}$ & $\begin{array}{l}\text { Estudo } \\
\text { seccional, } \\
\text { descritivo, } \\
\text { quantitativo }\end{array}$ & \begin{tabular}{llr} 
A maioria dos & \multicolumn{2}{c}{ Enfermeiros } \\
apresenta & nível & de \\
conhecimento inferior & ao \\
desejado em & relação aos \\
cuidados com feridas.
\end{tabular} \\
\hline
\end{tabular}




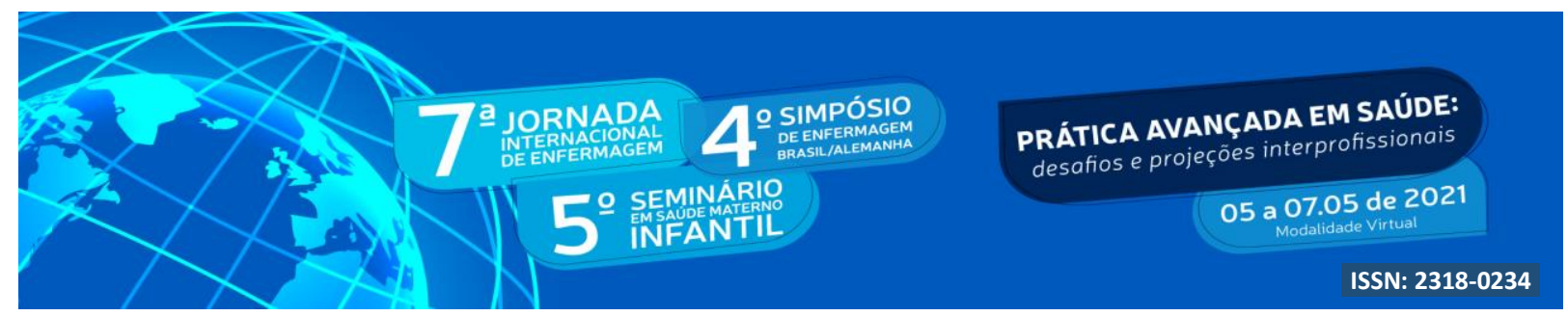

\begin{tabular}{|c|c|c|c|c|}
\hline $\mathbf{E}$ & $\begin{array}{l}\text { LÚCIO, F.D, } \\
\text { POLETTI NAA. }\end{array}$ & $\begin{array}{l}\text { Refletir sobre a importância } \\
\text { da utilização de exames } \\
\text { microbiológicos como } \\
\text { parâmetros } \\
\text { profissionais Enfermeiros } \\
\text { para o tratamento de } \\
\text { feridas. }\end{array}$ & $\begin{array}{l}\text { Descritivo } \\
\text { desenvolvido } \\
\text { sob a forma } \\
\text { de resenha. }\end{array}$ & $\begin{array}{l}\text { Os Enfermeiros precisam } \\
\text { desenvolver mais autonomia, } \\
\text { aperfeiçoar tratamentos } \\
\text { contribuir para a redução de } \\
\text { atrasos no processo cicatricial } \\
\text { das feridas. }\end{array}$ \\
\hline $\mathbf{F}$ & $\begin{array}{l}\text { CARDOSO, } \\
\text { D.D.S., } \\
\text { CARVALHO, } \\
\text { F.M.O., ROCHA, } \\
\text { G.B., MENDES, } \\
\text { J.R., CARDOSO, } \\
\text { S.D.B, ROCHA, } \\
\text { F.C.V. }\end{array}$ & \begin{tabular}{l} 
Conhecimentos \\
Enfermeiros \\
classificação de prevenção \\
\multicolumn{2}{l}{ de lesão por pressão. }
\end{tabular} & $\begin{array}{l}\text { Descritiva e } \\
\text { exploratória. }\end{array}$ & $\begin{array}{l}\text { A maioria dos profissionais da } \\
\text { Enfermagem } \\
\text { conhecimento insatisfatório } \\
\text { sobre LPP. }\end{array}$ \\
\hline
\end{tabular}

Fonte: Dados da Pesquisa Narrativa

A partir do levantamento de dados que foram coletados, durante o estudo, pretendeuse conhecer quais as barreiras enfrentadas pelos Enfermeiros na melhor escolha para o tratamento de lesões de pele. O objeto do presente estudo é de suma importância, devido à observação cotidiana das dúvidas dos enfermeiros que prestam cuidados à pacientes portadores de feridas.

A partir da análise das pesquisas emergiu a Necessidade de discutir sobre a atualização e aperfeiçoamento dos profissionais enfermeiros para realizar cuidado e a escolha adequada da cobertura. Realizar o cuidado de feridas ${ }^{(\mathrm{A}, \mathrm{B}, \mathrm{C}, \mathrm{D})}$, é um desafio que requer conhecimento específico, habilidade e abordagem ampla. Na busca de qualidade da assistência os estudos dessa pesquisa, salientam que existem diversas alternativas quando abordamos o tema avaliação de lesões de pele, porém, observa-se a necessidade de conhecimento tanto fisiopatológico como bioquímico dos mecanismos de cicatrização e reparação tissular para tratar e cuidar de pacientes com lesões de pele.

Tendo em vista que o Enfermeiro confronta cenários diversificados, é compreensível que também se depare com inúmeras dificuldades e uma delas ${ }^{(\mathrm{B})}$, encontrada e relatada, é a 


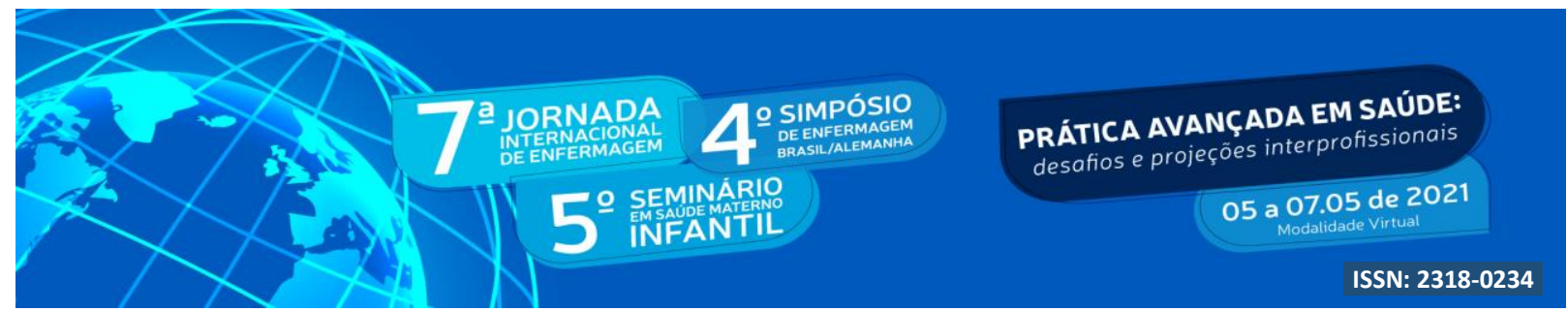

falta de atualização e aperfeiçoamento do próprios profissionais, em investir em educação permanente para permitir a troca de experiências vivenciadas institucionalmente no cuidado da pele com discussões de casos dos próprios pacientes que atendem no cotidiano, além das rodas de conversa na passagem de plantão, que foram mencionadas, como uma possibilidade para qualificar a atenção em saúde prestada pela equipe de Enfermagem das unidades de internação.

A Educação Permanente em Saúde (EPS) surge como estratégia de promoção do desenvolvimento dos processos de ensino-aprendizagem, centrando-se no sujeito que aprende, como agente ativo, autônomo e gestor de sua educação. A EPS é recente em nosso país, tornando-se política pública pela Portaria GM/MS no 198/2004, a qual instituiu a Política Nacional de Educação Permanente em Saúde (PNEPS) como estratégia em formação e desenvolvimento de trabalhadores de saúde, ela incorpora o aprender e o ensinar dentro da instituição, objetivando transformar as práticas profissionais para uma melhor qualidade no serviço, trazendo como perspectiva o aprender a partir dos problemas vivenciados pelos profissionais na prática e das necessidades dos pacientes.

Frente a isso pode-se dizer que a vontade por aprender e se atualizar nos tratamentos inovadores e terapêuticos e suas coberturas com diversas tecnologias ${ }^{(\mathrm{B}, \mathrm{C})}$, também deveria partir do profissional e seus interesses pelo tema aqui descrito. Os avanços tecnológicos nos têm possibilitado a utilização destes produtos que, evidentemente, aceleram a cicatrização das feridas e facilitam em muito a vida do paciente (FRANCO E GONÇALVES, 2008).

De acordo com a literatura, vê-se que o enfermeiro necessita ter não só a habilidade técnica, mas também deter o saber científico que envolve o seu fazer para decidir qual o melhor produto a ser utilizado ${ }^{(\mathrm{A}, \mathrm{B}, \mathrm{C}, \mathrm{E})}$ afinal o processo cicatricial é complexo e envolve diversos estágios fisiológicos, interdependentes e simultâneos, e é preciso saber reconhecê-los para planejar uma assistência adequada ao paciente de forma integrada. Com isso, os autores $^{(B, C, F)}$ reforçam a ideia de que é fundamental uma acurada avaliação da lesão, identificando-se cuidadosamente o estágio do processo cicatricial e tomando-se a decisão a partir dessa avaliação.

O enfermeiro precisa saber que o objetivo principal do curativo é o de manter um ambiente 


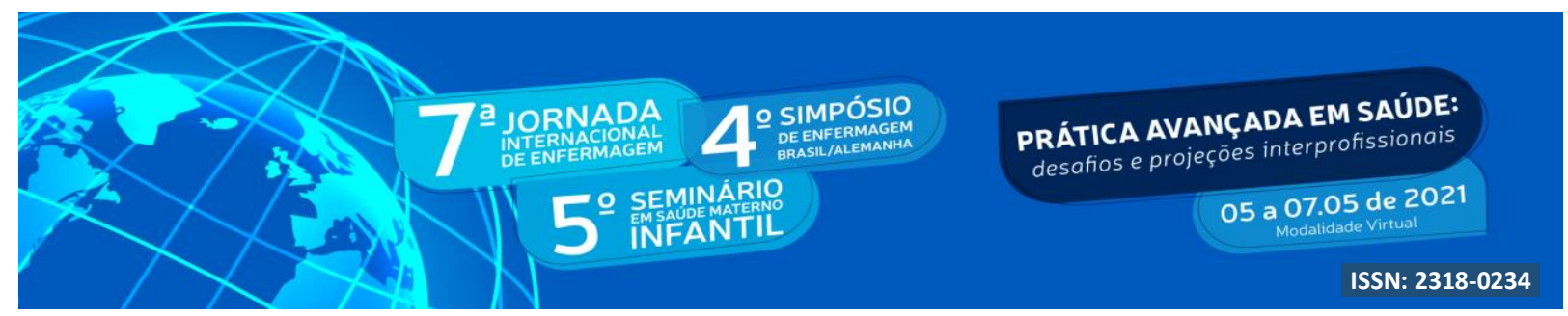

adequado para a cicatrização, através da avaliação da lesão, assim como realizar a limpeza e a cobertura de acordo com as técnicas assépticas, sustentadas ${ }^{(\mathrm{E})}$ em evidências científicas para otimização dos recursos humanos disponíveis e a redução de custos para instituição. Santos, 2014 salienta que a SAE é uma metodologia de planejamento, organização e execução de ações sistematizadas, que são realizadas pela equipe de enfermagem durante o tempo em que o indivíduo se encontra sob a assistência de enfermagem.

Em relação aos produtos que podem ser utilizados para o tratamento das feridas existem no mercado produtos desenvolvidos com alta tecnologia. Atualmente, a variedade de coberturas é cada vez maior e a pressão da indústria farmacêutica para ocupar espaço no mercado não para de crescer. Ainda não existem curativos ideais para tratar toda e qualquer ferida, porém um arsenal terapêutico vasto capaz de auxiliar o reparo tecidual em várias situações já é uma realidade. Cobertura trata-se de um meio terapêutico que consiste na limpeza e/ou aplicação de material sobre uma ferida podendo ser para sua proteção, absorção e drenagem, com a finalidade de melhorar as condições do leito da ferida e sua resolução. Curativos podem ser, em algumas ocasiões, o próprio tratamento definitivo ou em outras, apenas uma etapa intermediária para o tratamento cirúrgico (ISAAC et al, 2010).

Além de deter o conhecimento para o tratamento de lesões, o enfermeiro precisa saber prevenir que elas venham acontecer, para isso ele precisa ter domínio sobre os fatores que podem influenciar no aparecimento delas e saber de estratégias para preveni-las como o uso de instrumentos específicos, que temos como exemplo a escala de $\operatorname{Braden}^{(\mathrm{C}, \mathrm{D}, \mathrm{F})}$. A lesão por pressão pode ser evitada com o conhecimento dos fatores associados ao seu processo de formação desde que seja detectado o paciente com potencial de risco para esse tipo de lesão.

Tendo em vista as barreiras encontradas, para uma avaliação integral do indivíduo, não somente da ferida, é fundamental a utilização de instrumentos que lhes fornecerão subsídios no tratamento de feridas e a escolha do agente terapêutico adequado, além de possibilitar o acompanhamento da evolução da lesão durante todo o processo de reparação tecidual em cada fase da cicatrização (ESPÍRITO SANTO et al., 2013).

\section{CONCLUSÃO}

Essa pesquisa atendeu ao seu objetivo principal que é analisar na produção cientifica, 


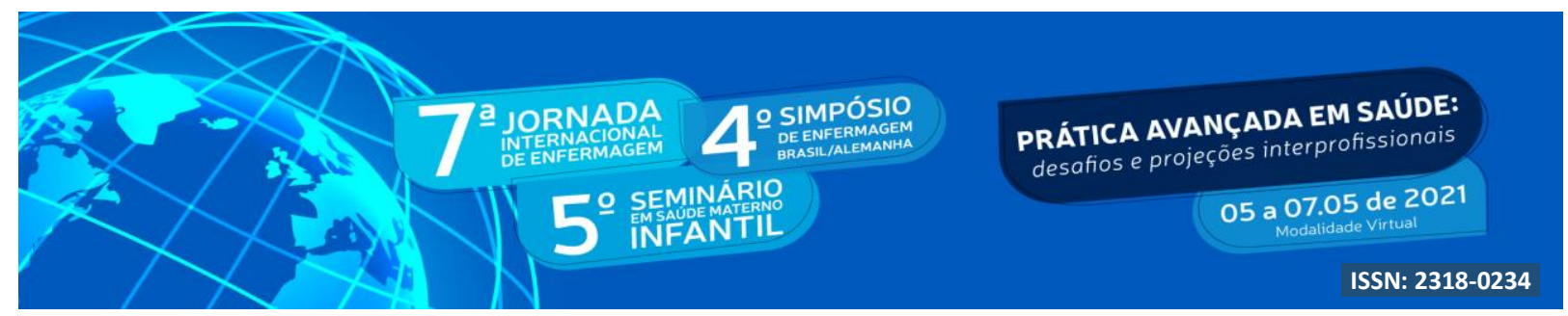

quais as barreiras encontradas pelos enfermeiros na hora da escolha da melhor conduta no tratamento de lesões. A análise da literatura demonstrou que dentre os desafios na atuação do enfermeiro, destaca-se a mnecessidade de atualização e aperfeiçoamento dos profissionais para realizar cuidado e a escolha adequada da cobertura.

Pelo seu caráter articulador e integrador, o Enfermeiro possui habilidades e competências técnico - cientificas para exercer o cuidado com lesões, tendo um papel de grande importância para garantia de um cuidado integral do indivíduo e desempenha um trabalho de extrema relevância tratando-se de feridas, já que tem maior contato com o paciente.

O Enfermeiro deve ter empoderamento e conhecimento para que seja escolhido o melhor tratamento para o seu paciente, para isso ele precisa ter domínio sobre os fatores que podem influenciar no aparecimento ou no agravo delas e saber de estratégias para preveni-las ou amenizá-las como o uso de instrumentos específicos. Sendo assim, reafirma-se a importância de desenvolver estudos sobre a percepção e conhecimento dos enfermeiros sobre prevenção e tratamento de feridas.

\section{REFERÊNCIAS}

ABBADE, L. P. F. Preparo do leito da ferida. Malagutti W. Curativos, Estomias e Dermatologia: uma abordagem profissional. São Paulo: Martinari, p. 63-76, 2010.

BRASIL. Ministério da Saúde. Portaria n⿳ 825, de 25 de abril de 2016. Redefine a Atenção Domiciliar no âmbito do Sistema Único de Saúde (SUS) e atualiza as equipes habilitadas. Brasília, DF: Ministério da saúde, 2016.

BRUM, M. L. B. et al. Protocolo de assistência de enfermagem a pessoa com feridas como instrumento para autonomia profissional. Rev Enferm UFSM, V. 5, n.1, p.50-57, 2015.

CHIBANTE, C. L. P.; SANTO, F. H. E.; SANTOS, T. D. Perfil de clientes hospitalizados com lesões cutâneas. Rev. cuba. Enferm, V. 31, n.4, 2015.

COFEN-Conselho Federal de Enfermagem. Resolução COFEN-0501/2015. Norma técnica que regulamenta a competência da equipe de enfermagem no cuidado às feridas e dá outras providências [Internet]. Brasília: COFEN, 2015.

CROSSETTI, M. G. O. Revisão integrativa de pesquisa na enfermagem o rigor cientifico que lhe é exigido; Rev Gaúcha Enferm, p.8-9, 2012. 


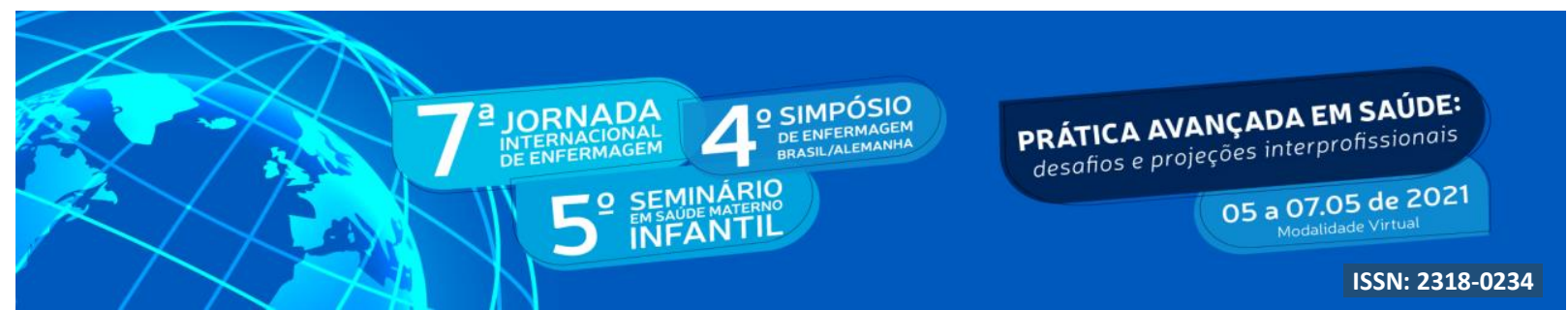

ESPÍRITO SANTO, P. F. et al. Uso da ferramenta Pressure Ulcer Scale for Healing para avaliar a cicatrização de úlcera crônica de perna. Revista Brasileira de Cirurgia Plástica, v. 28, n. 1, p. 133-141, 2013.

FARIA, G. B. G. et al. Conhecimento e prática dos enfermeiros sobre o cuidado com feridas. Rev enferm UFPE online., V.10, n.12, p.4532-8, 2016.

FERREIRA, A. M., CANDIDO, M. C. F. S., CANDIDO, M. A. O cuidado de pacientes com feridas e a construção da autonomia do enfermeiro. Rev enferm. UERJ, V.18, n.4, p.656-60, 2010.

ISAAC, C., et al. Processo de cura das feridas: cicatrização fisiológica. Rev Med. V.89, n.3/4, p. $125-31,2010$

JACONDINO, C.B. et al,. Educação em serviço: qualificação da equipe de enfermagem para o tratamento de feridas. Cogitare Enferm V. 15, n.2, p.314-8, 2010.

KRAEMER, F. Z.; DUARTE, M. L. C.; KAISER, D. E. Autonomia e trabalho do enfermeiro. Rev Gaúcha Enferm., V32, n.3, p.487-94, 2011.

MINAYO, M. C. S. O desafio do conhecimento: pesquisa qualitativa em saúde. São Paulo: HUCITEC, 2000.

MONTANHA, D., PEDUZZI. M. Permanent education in nursing: survey to identify the necessities and the expected results based on the workers conception. Rev Esc Enferm USP, V.44, n3, p. 597-604, 2016.

OLIVEIRA, F.P., et al. Nursing interventions and outcomes classifications in patients with wounds: cross-mapping. Revista gaucha de enfermagem, V. 37, n. 2, 2016.

SANTOS, W. N. Sistematização da assistência de enfermagem: o contexto histórico, o processo e obstáculos da implantação. J Manag Prim Health Care, v. 5, n. 2, p. 153-158. 2014.

SANTOS, E. I. et al. Autonomia profissional e enfermagem: representações de profissionais de saúde. Rev Gaúcha Enferm. Vol.38 no.1 Porto Alegre 2017 Epub May 18, 2017.

SEHNEM, G. D. et al. Dificuldades enfrentadas pelos enfermeiros no cuidado de enfermagem a indivíduos portadores de feridas. Cienc Cuid Saude V. 14, n.1, p.839-846, 2015.

SMANIOTTO, P.H.S.; FERREIRA, M.C.; ISAAC, C.; GALLI, R. Sistematização de curativos para o tratamento clínico das feridas. Rev Bras Cir Plást.V.27, n.4, p.623-6, 2012.

SOUZA, Pedro Henrique de. et al. Sistematização de curativos para o tratamento clínico das feridas. Rev Bras Cir Plást.V.27, n.4, p.623-6, 2012 